\title{
On the Fundamental Meaning of the Principle of Least Action and Consequences for a "Dynamic" Quantum Physics
}

\author{
Helmut Tributsch \\ Bio-Mimetics in Energy Systems Program, Carinthia University for Applied Sciences, Villach, Austria \\ Email: helmut.tributsch@alice.it
}

Received 12 December 2015; accepted 23 February 2016; published 26 February 2016

Copyright (C) 2016 by author and Scientific Research Publishing Inc.

This work is licensed under the Creative Commons Attribution International License (CC BY). http://creativecommons.org/licenses/by/4.0/

(c) (i) Open Access

\section{Abstract}

The principle of least action, which has so successfully been applied to diverse fields of physics looks back at three centuries of philosophical and mathematical discussions and controversies. They could not explain why nature is applying the principle and why scalar energy quantities succeed in describing dynamic motion. When the least action integral is subdivided into infinitesimal small sections each one has to maintain the ability to minimize. This however has the mathematical consequence that the Lagrange function at a given point of the trajectory, the dynamic, available energy generating motion, must itself have a fundamental property to minimize. Since a scalar quantity, a pure number, cannot do that, energy must fundamentally be dynamic and time oriented for a consistent understanding. It must have vectorial properties in aiming at a decrease of free energy per state (which would also allow derivation of the second law of thermodynamics). Present physics is ignoring that and applying variation calculus as a formal mathematical tool to impose a minimization of scalar assumed energy quantities for obtaining dynamic motion. When, however, the dynamic property of energy is taken seriously it is fundamental and has also to be applied to quantum processes. A consequence is that particle and wave are not equivalent, but the wave (distributed energy) follows from the first (concentrated energy). Information, provided from the beginning, an information self-image of matter, is additionally needed to recreate the particle from the wave, shaping a "dynamic" particle-wave duality. It is shown that this new concept of a "dynamic" quantum state rationally explains quantization, the double slit experiment and quantum correlation, which has not been possible before. Some more general considerations on the link between quantum processes, gravitation and cosmological phenomena are also advanced.

\section{Keywords}

Least Action, Calculus of Variations, Dynamic Energy, Quantum Paradoxes, Quantum Correlation 


\section{Introduction}

Action means that the energy involved in making a change is multiplied over the time during which the change occurs. The function, which is integrated over time to obtain action $S$ (Hamilton's first principal function)

$$
S=\int_{t_{0}}^{t_{1}} L \mathrm{~d} t=\int_{t_{0}}^{t_{1}}(K-U) \mathrm{d} t
$$

is the well known Lagrangian function $L$ (with $K=$ kinetic energy and $U=$ potential energy). It depends on location, velocity and time: $L=L(q, \dot{q}, t)$ and describes and summarizes the dynamics of a system. The Lagrangian can be plotted against time. The area below is action. It is well known that when the Lagrangian function is available, it can be introduced into the Euler-Lagrange equation to obtain the equations of motion. It explains how the minimization takes place:

$$
\frac{\partial L}{\partial q}-\frac{\mathrm{d}}{\mathrm{d} t} \frac{\partial L}{\partial \dot{q}}=0 \quad \text { Euler-Lagrange equation }
$$

The principle of least action has not only been applied to derive the dynamic behavior of systems in gravitational fields, but also in quantum mechanics and space-time environments. The term "least" means here that the first order change in the value of action is zero [1] [2].

It is not surprising that the search for a formula describing the universe has been mostly the search for an ultimate Lagrangian. From that it can be concluded that the principle of least action is recognized as a key element towards the fundamental understanding of nature.

Understanding the fundamental meaning of the related principle of least action promises to be an important step towards a deeper understanding of nature. But today it can still safely be said, that nobody really knows why nature applies the principle of least action and what it fundamentally means. To find an answer and to test consequences for quantum physics is the aim of this publication.

\section{Historical Background}

During the last three centuries, no other principle has nourished hopes into a universal theory, has constantly been plagued by mathematical challenges, and has ignited metaphysical controversies about causality and teleology more than did the principle of least action [3]. It goes back to Maupertius in the mid-eighteenth century and was preceded by Fermat's principle of least time from the seventeenth century.

Traditionally, the principle of least action has been thought to have a deeper philosophical significance because it seems to suggest that physical systems are governed by final causes, or that the cause of something has the character of a final cause. The principle of least action was therefore connected with teleology, which contends that natural phenomena have intrinsic purposes. Why is the principle of least action functioning this way?

Maupertius saw in the principle of least action "the grand scheme of the universe". Leibniz recognized a principle of determination derivable from maxima and minima, such as done in the principle of least action [4]. For Helmholtz the principle of least action became a "leitmotif" for formulating new laws [5]. Planck considered the principle of least action as a significant step towards the aim of attaining knowledge about the real world [6]. He concluded that among the achievements of physical science the principle of least action comes closest to the final goal of theoretical research. Even Einstein came to the conclusion that the principle had to be an essential element in his general theory of relativity [7].

The variational calculus itself, which helps to find the minimum of the action integral (1) over a scalar Lagrangian energy quantity (L), has also been repeatedly discussed in a controversial way with important mathematicians involved such as Gauß, Jacobi and Hilbert. Weierstraß in the 19th century finally introduced relevant improvements.

\section{Results}

\subsection{The Principle of Least Action Is a Dynamic Statement on Energy}

The principle of least action selects, at least for conservative systems, where all forces can be derived from a potential, the path, which is also satisfying Newton's laws, as for example, demonstrated by Feynman [8] via the 
calculus of variations.

By performing it, it is recognized that least action is only satisfied when Newton's laws are valid. However, the second law of thermodynamics cannot be derived from Newton's laws, and consequently not from the principle of least action as it is handled and understood now.

How can the principle of least action be understood in more detail? The path, described by the least action integral, can be split up in many infinitesimally small sections, which equally have to follow the principle of least action. We replace the Lagrange function $L$, which expresses the dynamically available energy, which generates motion, by a more general energy quantity $E$, which is dependent on time, position and velocity, since $L$ in physics is treated as a scalar quantity only. Then one obtains for an infinitesimal section of the action integral (variables: position, time):

$$
S=\lim _{\Delta t, \Delta q \rightarrow 0} \int_{t}^{t+\Delta t} \int_{q}^{q+\Delta q} E(t, q, \dot{q}) \mathrm{d} t \mathrm{~d} q \approx E(t, q, \dot{q}) \Delta t \Delta q
$$

Its ability to minimize as an infinitesimally small section is a mathematical necessity, if the principle of least action should be generally valid (Feynman). Mathematically this is entirely clear, since a deviation from such a condition for only one infinitesimal section would violate the principle of least action in general. The derivative with respect to time and location has consequently to become a minimum, approaching zero.

$$
\mathrm{d} S^{\prime}=\frac{\mathrm{d} S}{\mathrm{~d} t \mathrm{~d} q} \approx \frac{E\left(q, q^{\prime}, t\right) \Delta t \Delta q}{\mathrm{~d} t \mathrm{~d} q} \approx E\left(q, q^{\prime}, t\right) \rightarrow \min
$$

The behavior of the infinitesimal action remains merely determined by the energy quantity $E(t, q, \dot{q})$. This energy has to have the property to decrease and minimize. What does it now mean when the energy, which produces movement, has to approach a minimum. What are the possible interpretations for this minimization process?

Feynman comes to the conclusion that the differential statement on the path of least action can only concern the derivatives of the potential, that is the force at a point. He speculates that the particle "looks" at all possible trajectories before selecting the one subject to least action.

The interpretation given here is different and definitively simpler. Relation (4) clearly shows that the dynamic energy quantity $E(t, q, \dot{q})$ has to have itself the ability to minimize. It is important now to recall first what this energy means. It is definitively not the total energy of the system, which would be the sum of kinetic and potential energy $(K+U)$. It would anyway stay constant during the dynamic process, which is subject to the minimization of least action, while energy is being converted and entropy generated. The Lagrange function is the difference of kinetic and potential energy $(L=K-U)$. It describes the energy, which becomes available for generating dynamic motion. It is the free energy, which can be converted into other energy forms and into not any more available (entropic) energy. This free energy can of course and also must decrease during an energy conversion process.

Now it becomes clear why the Lagrange function $L$ is, in this publication, replaced by a generalized energy $E$. The Lagrange function in classical physics is defined as a scalar quantity, a number. A number has no tendency or ability to minimize. It is defined to act as a mere number. This means that the condition that an infinitesimal segment of action, as described in (3), is minimized, cannot be fulfilled. It is important to point out that the present physical formalism does not respect this consequence. Free energy, as considered in the Lagrange function L, which is generating motion, is handled as a scalar quantity. Scalar energy, energy as it is understood in physics now, is defined to have the ability to work, but no interest. Nevertheless an apparent solution was found. It is variational calculus. In fact, variational calculus is imposing and simulating a variation, which a scalar quantity itself cannot perform. This enables the consequence that the properties of the principle of least action can at least partially and superficially be simulated and exploited. Why is physics doing that?

All fundamental physical laws are formulated in such a way as to function in both positive and negative time direction. There is now no fundamental law in physics claiming a preferred time direction, as the here discussed conditions (3) and (4) do. The entropic time arrow of present physics can only be derived from a time invertible statistical ensemble by drastically simplifying the mathematical procedure (Boltzmann's H-Theorem, coarse graining procedures), which means by throwing away information. Information, however, has an energy content. This energy is thrown away, which explains why the system cannot any more return to the beginning, but is then 
proceeding in one direction only (the same mathematics could also allow the function to proceed into opposite time direction, which is not observed). The derived function can, for this obvious condition, not recover the original situation. The statistical time direction is thus just manipulated mathematically and would anyway not work where self-organization and local reduction of entropy takes place.

And energy, which is powering changes, is in today's physics, imagined as a scalar quantity without relation to change. Einstein himself compared our understanding of energy with a beggar, who actually is a millionaire, but nobody knows and sees it. This situation is strange, because our concept of energy did evolve from efforts, since antiquity, to understand change. Therefore the question was asked by philosophers and early scientists as to something, which remains conserved within all changes around us. What remains conserved turned out to be energy. However, during the development and optimization of the energy concept in the course of the 19th century energy lost its relation to changes and irreversibility and became a scalar. It is now a quantity, which is just a number, without any relation to change.

In contrast, it is known that all changes $(C)$ are originating from conversion of energy $(E)$. Changes must consequently be a function of energy. Mathematically also the inverse relation must therefore hold:

$$
C=f(E) \text { and } E=f(C)
$$

It essentially leads to the same conclusion as to be drawn from relation (4): Energy has an inherent property related to change (provided the constraints of the system allow that). Such a property is today however not recognized. Energy, as handled today, is just a scalar quantity, a number, without relation to change.

But the infinitesimal section of action (3) must necessarily express the ability to minimize. It must be able to decrease, which means that the energy $E(t, q, \dot{q})$ must be able to decrease its presence in this state (relation (4)). The process is time oriented. When every point on the track of a stone rolling down a hill minimizes the presence of energy per state, a minimum action route will automatically result.

It is consequently claimed here, that available energy is fundamentally time oriented and aims at decreasing its presence per state. This means a paradigm change, since a time orientation is fundamentally imposed. This explains why action is indeed minimized. It is minimized because energy has the drive to minimize its presence per state. Thereby waste energy in not usable form is generated and entropy increases. The second law of thermodynamics follows immediately, which is an important result, because it cannot be deduced from the present day time-invertible physical formalism.

We can, of course, imagine, that the number, the scalar named Lagrange function, can vary and minimize. This is actually done via the variational calculus to find the equations of motion. We are simply pretending that a number has the tendency to minimize. But nature does not care about our imagination. A stone rolls down a hill anyway following the principle of least action. And this can only happen when a law exists in which an infinitesimal action segment (3) has the property to minimize. In other words, the energy available for motion $E(t, q, \dot{q})$ cannot be a scalar, it must have time oriented properties. If the principle of least action is considered to be fundamentally relevant for physics, the definition for free energy must consequently change:

"free energy has the tendency to decrease and minimize its presence per state, within the restraints of the system"

It is here suggested that the principle of least action is nothing else than the statement that our world is fundamentally time oriented and irreversible. Rate controlling entropy production is critically and fundamentally shaping the change in our environment and determining the progress of time.

How did the energy concept in present-day physics loose its relation to change, from where it was actually born? The Italian-French mathematician J.-L. Lagrange, around 1788, when studying his famous energy equations for dynamic systems, still considered and investigated conditions, which reflected irreversibility and time orientation. This means, he paid attention to change. Also the Irish mathematician W.R. Hamilton, when, during the first half of the 19th century, deriving the now famous Hamilton functions, still argued that external irreversibility should be considered. He also felt that there had to be a relation to change. Other scientists also had the impression that the principle of least action is related to change. Ernst Mach [9], for example, concluded, that "the principle of 'vis viva' (energy of movement) is the real foundation of the theorem of least action". With Jacobi [10] together they recognized the meaning of the principle of least action in the least expenditure of work. Energy in present day physics, in fact, got the capacity to do work, but has no interest to do it and no preference to minimize, to decrease the presence of energy per state. The principle of least action ended up in that scalar 
energy is treated with variational calculus to predict motion. This gives the superficial illusion that the principle of least action is compatible with time-reversibility. But it is not. It is a statement on time-orientation and was for that reason linked to teleology, which is always time oriented. Historically, the question was asked how a system can know in advance via what path it can minimize action. Relation (3) and (4), which describe minimization of an infinitesimally small interval of action, give a precise answer: it is sufficient to assume that free energy is time oriented, that it minimizes its presence per state. It will do that for any point of its path, subject to the constraints given, and thus find that path which is subject to a minimum of action. A fundamentally directed and irreversible nature of energetic behavior in dynamic processes is the answer to the strange philosophical mystery around the principle of least action. Energy must have a relation to change, as also deducible from consideration (5).

\subsection{Consequences for Quantum Physics}

When the conclusions drawn are reasonable, they can be tested. Quantum physics with its counter-intuitive aspects was selected for an intellectual experiment. Quantum states are presently defined as equilibrium states. What happens when they are considered fundamentally dynamic? When dealing with particle and wave in quantum physics subject to a dynamic energy it is clear that they cannot be equivalent and simultaneous. The system with inferior energy value (inferior capacity to do work) will follow from that with higher free energy value. The system with inferior free energy must be the wave with spread out energy distribution (in conventional quantum physics energy in particle and wave is considered equivalent). However the overall reversible nature of energy behavior within the particle-wave quantum phenomenon (no energy is finally turned over) also has to be considered. Energy in a particle should be able to expand into a wave and it thereby also loses working ability while generating (a fundamental type of) entropic energy. The formalism for the quantum system, however, must therefore set aside energy in the form of information to guarantee reversibility in the absence of overall energy turnover. A kind of fundamental Maxwell demon is needed to bring back the energy distributed in a wave to the shape of the particle. Since this demon will need information, and thus energy to do so, this energy has to be set aside by the particle before converting into a wave. It has to be set aside to act from the outside of the expanding energy system.

The energy of the particle and of the wave should therefore not be identical and should not ignore space, as seen in Figure 1(a) for conventional quantum physics, but the energy of particle $E_{p}$ should be equivalent to the distributed energy in the wave $\sum_{w} E_{w}$ (describing the sum over all $w$ tiny energy particles) plus the fraction of energy which is no longer available for work $E_{e}$ due to the expansion in space, and contributing to entropy, plus the "negentropic" energy $E_{n}$ (energy in the form of information), which would be required to make energy conversion reversible (see Figure 1(b)). This would work via a fundamental Maxwell demon. He uses information with energy content $E_{n}$ to handle the energetic tasks for the recovery of the distributed energy in the wave to reshape the original particle with energy $E_{p}(h=$ Planck constant of action, $v=$ light frequency):

$$
h v=E_{p} \leftrightarrow \sum_{w} E_{w}+E_{e}+E_{n}
$$

In other words: the energy in the particle $E_{p}$ converts into the distributed energy of the wave $\sum_{w} E_{w}$ plus the non-usable energy in the form of entropy $E_{e}$ plus energy $E_{n}$ set aside in the form of information needed for the reconversion into the particle. The latter energy $E_{n}$ is a kind of "information self-image of matter". No energy is exchanged with the outside and the energy of information, which is set aside from the beginning, is tailored in such a way that the total energy as expressed in formula (7) is sustained. We have thus permitted that the energy converted from a particle into a wave loses some ability to perform work (it assumes a microscopic form of entropic energy), but simultaneously provides energy in the form of information (also called negentropic energy because it behaves and acts somehow in the opposite way to entropic energy), which subsequently reconverts the entropic energy. The information provided by a hypothetical microscopic Maxwell demon is thus used to re-concentrate the energy into a particle (Figure 1(b)). This does not contradict the second law of thermodynamics, which states that the entropy increases in a closed space or volume, because information is assumed to come from outside. The system works as it does with information provided to a three dimensional printer, which 
a)

traditional model

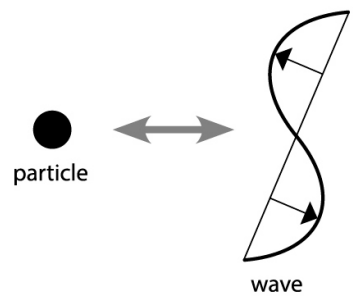

b)

new model
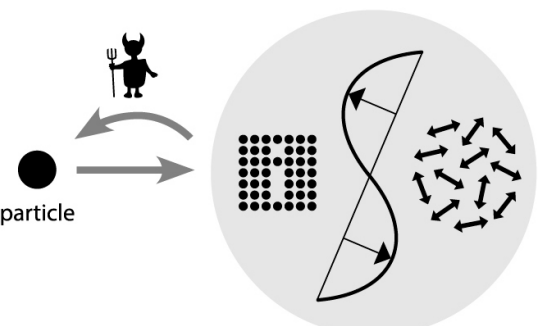

information self-image
+ wave + entropy

Figure 1. The particle-wave duality in conventional quantum physics (a) and within the dynamic energy model (b) in which information on matter is involved in the backformation of the particle from the wave.

assembles a three-dimensional object via pure information. This is required to provide reversibility of particlewave inter-conversion within the particle-wave duality in a fundamentally oriented world. When the particle contains sufficient energy to account for the information needed for reconstruction, then the back conversion from a state of distributed energy plus the accompanying entropic energy can indeed proceed according to relation (7). We will now proceed to explain diverse quantum phenomena using the proposed new symbolism (Figure $1(b))$.

\subsubsection{Quantization}

When an electron selects an orbit around a nucleus of an atom, it aims at minimizing its energy per state while also forming a wave. Considering relation (7) this means that also the energy needed for information $\left(E_{n}\right.$ in relation (7)) has to be minimized. As a consequence only the simplest wave patterns around the atom, corresponding to a minimum of information for describing them, will be selected. This is quantization (Figure 2). It explains why the circulating electron does not leave a quantized orbit gradually while losing energy through radiation, as classically expected. This would simply require an increase in $E_{n}$, the energy in form of information, and contradict statement (6).

\subsubsection{The Double Slit Experiment}

Classical particles select a path following the principle of least action. For quantum systems, in contrast, it was found that they seem to consider all possible paths towards the final destination (Richard Feynman's sum-overpaths method). In the case of the double-slit experiment (Figure 3) an individual particle would select paths through both slits. Such a behaviour, a double path for one particle, is irrational, but can be calculated and indeed explains the observed patterns produced on the screen. How can such a sum-over-path phenomenon be reinterpreted in the light of the proposed alternative quantum model? A particle would start following the principle of least action. Then it would change into a wave, equivalent to many distributed tiny energy dots (according to (7) and Figure 1(b)). Each of these would continue following the principle of least action and cross through one of both slits. Behind these, the tiny energy dots would regroup (according to relation (7)) to form a particle again-a particle which, of course, has "seen" both slits. The two-slit experiment becomes rationally understandable. This is due to the information self-image of matter, which is proposed to link particle and wave.

\subsubsection{Quantum Correlation}

When particles split up yielding quantum correlation, the information on their particle-wave state $\left(E_{n}\right.$ in relation (7)) will also have to split up. But some common information structure must be maintained if required by conservation laws (e.g. spin conservation). Where should this information then be deposited? It can be imagined that it is this joint information, which keeps the mysterious, "spooky" correlation contact (Figure 4). One may compare this situation with that of two people, who separate, but maintain an exchange of information via cellular phones. There is no irrational phenomenon behind. 

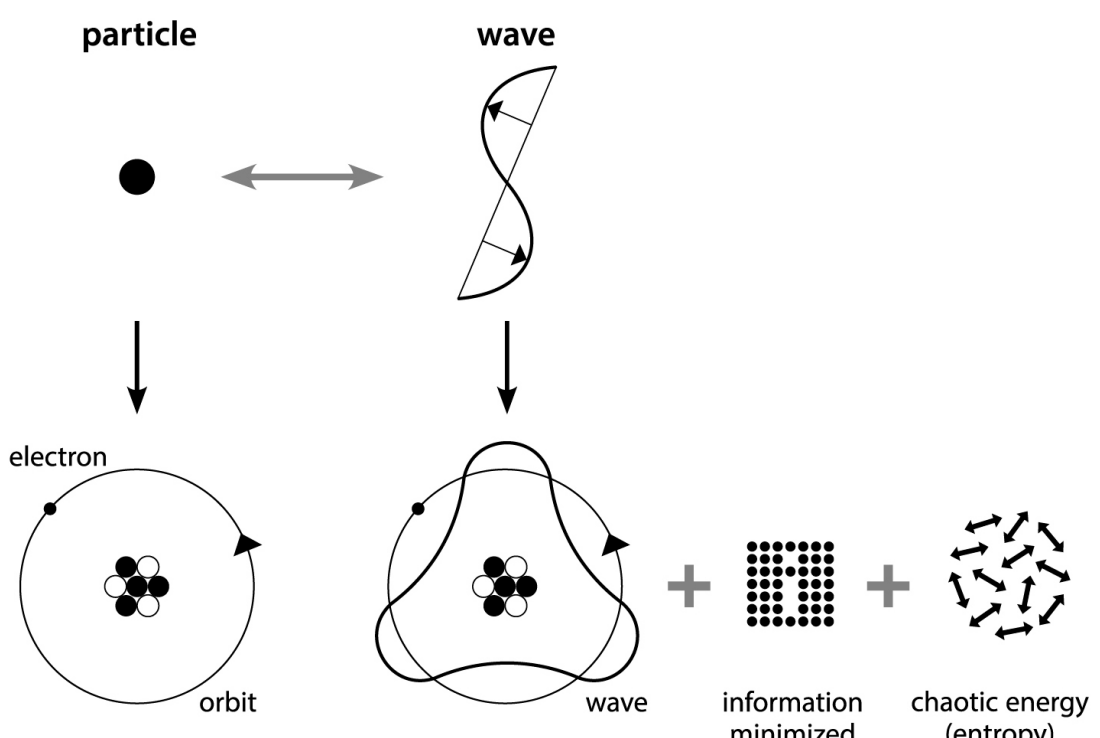

Figure 2. A dynamic energy concept of the quantum state involves information on matter, which equally has to be minimized, leading to the selection of the simplest wave patterns around a nucleus. This is quantization.
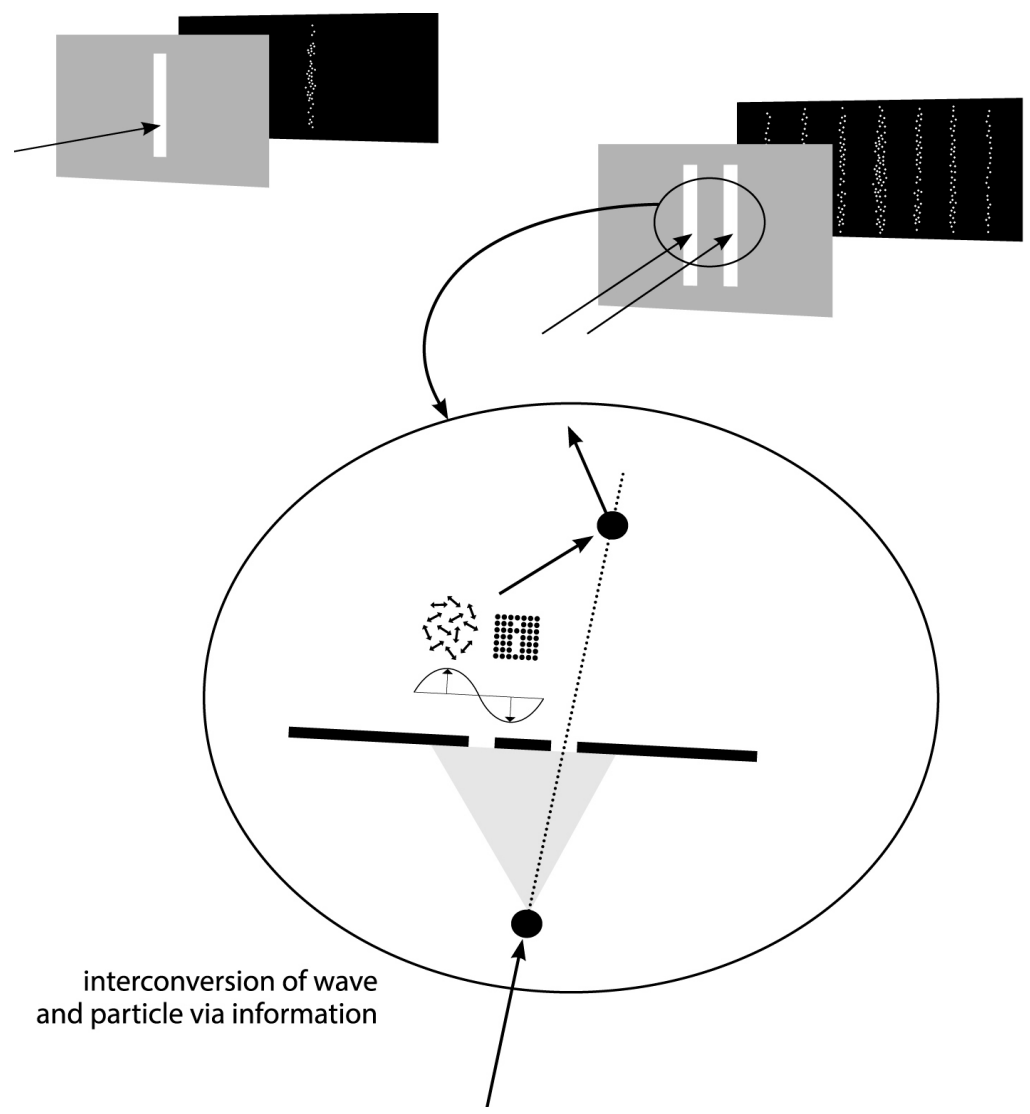

Figure 3. The dynamic inter-conversion of particle and wave involving a self-image of information allows a rational understanding of the double slit experiment without assuming non-locality properties. Also individual particles convert into a wave and reconvert into a particle (with the memory of the second slit) via the self-image of information. 


\section{original particle quantum correlated}

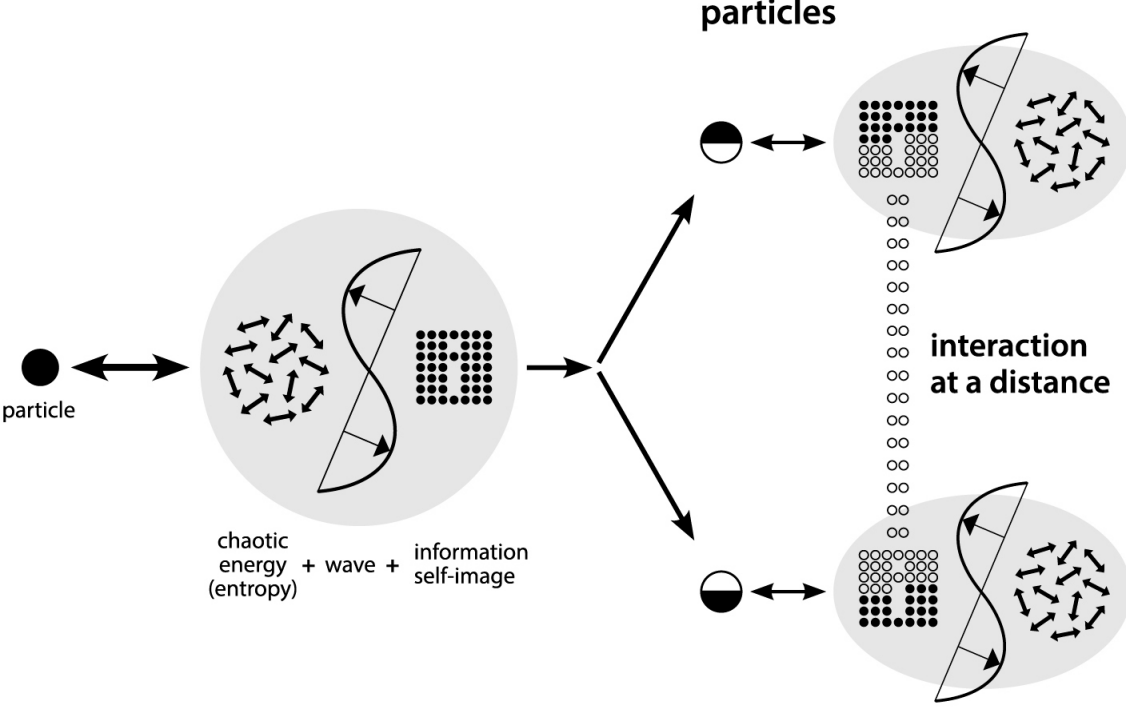

Figure 4. The fact that information had to be involved in the dynamic particle-wave duality (Figure 1(b)) leads to a rational explanation of quantum correlation, of interaction at a distance. It is simply the portion of the already present self-image of information, which cannot easily be divided up between separating particles because of conservation laws. One gets the impression that quantum phenomena can be rationally understood.

\section{Discussion}

The principle of least action has been applied in science as a basic tool for shaping different disciplines and it has been a guideline in the up to now futile search for a world formula. The variational calculus applied to a scalar driving energy replaced historically the property of a time oriented, vectorial free energy mathematically identified here. This way it was still possible to partially exploit the extraordinary property of this principle in giving answers to fundamental questions in physics. The only superficially correct historically identified approach, however, obscured the real meaning of the principle of least action. It says that the world is fundamentally time oriented and irreversible. The conventional approach thus provided the link to the time invertible Newton's equations and to a time invertible world of physics, but it denied the derivation of the second law of thermodynamics and possibly additional insight. The latter is feasible when, as shown here, the dynamic property of energy is taken seriously. The second law of thermodynamics immediately follows since in a closed system maximum entropy will result from statement (6). This is an important result of present considerations and not in conflict with classical ways of handling physics, which can be tolerated as a limiting case.

The identification of a fundamental time orientation in physical processes has the consequence, that an aimed energy (and oriented time) has also to be applied on elementary, atomic, and molecular level. The world becomes fundamentally irreversible also in the domain of quantum physics. What are the consequences? Dynamic energy aims at implementing changes. It turns out that particle and wave are not equivalent any more, but that the second follows from the first. Most important, its spread energy is also less "valuable" since it will have a decreased potential for doing work (this is differently understood in conventional quantum physics, where particle and wave are energetically seen to be equivalent). As a consequence it must be considered that a kind of fundamental entropy is generated ( $E_{e}$, symbolically shown in Figure 1(b)). Energy of information on the state of matter, an information self-image of matter, is therefore needed to recover the particle from the wave form and has to be included in the description of quantum processes (relation (7) and Figure 1(b)). It has to be set aside, while a particle is being formed from a wave. It turns out that for understanding quantum processes rationally both a description of matter and information on this matter is required. If this is done, cognitive difficulties and paradoxes of quantum physics disappear, as shown here with quantization, the double slit experiment and quantum correlation. According to the presented considerations the claim that irrationality and counter-intuitivity in quantum physics is a fundamental property of nature [11] has to be challenged. It appears that paradoxes and 
irrationalities are only present because the message to be drawn from the principle of least action, a fundamental orientation of energy, is not considered. A time-invertible physics is not providing the information needed for intuitive understanding of quantum processes.

The introduced information self-image of matter (expressed by $E_{n}$ in relation (7)) turned out to be a key for introducing rationality into quantum phenomena. It is interesting to note, that the here identified missing information in conventional quantum mechanics, which generates paradoxes and irrationality in understanding, would be information itself, the "information self-image of matter", $E_{n}$.

It may be asked now what experimental evidence could be given on information being involved in quantum states (Figure 1(b)) as defined by relation (7). Information has an energy content and should definitively be measurable. What can be detected to exist around particles and matter since information is claimed to be associated with any particle of matter (Figure 1(b))? What penetrates matter, increases with the amount of matter and is also present inside matter? There is convincing evidence that this is simply gravitation. Gravitation here would be neither a force at close range, as in general relativity, nor a force at a distance, like in Newton's theory. Gravitation here is information on matter with the task of implementing a minimization of energy per state. This minimization works, since matter is attracted. Gravitation would work like a system operated by remote control via transmitted information. A planet around the sun would circulate like remote controlled via gravitation towards minimizing action. As gravitation this information would equally be involved in quantum correlation (Figure 4), in quantization (Figure 2) and in diffraction (Figure 3), forming the link between cosmic and quantum phenomena. Information on matter, $E_{n}$, would thus have to be considered as a fundamental law enabling elementary fenomena. This is new for physics, but in fact not surprising, considering the enormous potential of information already visible in modern information technology. This technology is, in fact, functioning on the basis of natural laws. Why should nature herself not use them? It can be demonstrated that this information selfimage of matter also gives a straightforward access to understanding and explaining an always constant light velocity. This points at a much more reasonable and straightforward alternative to the presently counter intuitive fourdimensional space-time, designed to impose gravitation and the always constant light velocity via empty space.

There are, of course many consequences and questions to be deduced from such a new approach towards explaining fundamental processes. Last not least it has also to be clarified why understanding of quantum physics can be improved in spite of the Bell theorem, which essentially states that quantum physics is complete and paradoxes fundamental. This and other challenges and consequences are discussed in some detail in a parallel book publication [12].

The proposed paradigm change from a time invertible to a fundamentally time oriented world, a postulate derivable from the principle of least action, seems to promise the possibility of a return to a more rationally and more easily understandable quantum physics, while experimental results are not touched. It therefore deserves critical discussions and controversy.

\section{References}

[1] Lanczos, C. (1986). The Variational Principles of Mechanics. Dover Publications, New York. Yourgrau, W. and Mandelstam, S. (1968) Variational Principles in Dynamics and Quantum Theory. Pitman, London.

[2] Lemons, D.S. (1997) Perfect Form. Variational Principle, Methods, and Applications in Elementary Physics. Princeton University Press, Princeton, NJ.

[3] Stöltzner, M. (2002) The Principle of Least Action as the Logical Empiricist’s Shibboleth. http://philsci-archive.pitt.edu/616/; Terekhovich, V. Metaphysics of the Principle of Least Action. http://www.vtpapers.ru/Papers/MetaphysicsPLA.pdf

[4] Leibniz, G.W. (1697) The New Synthese Historical Library, 2, 486-491.

[5] Helmholtz, H.V. (1886) Über die physikalische Bedeutung des Princips der kleinsten Wirkung. In: Wissenschaftliche Abhandlungen, Leipzig, 1895, vol. III, 203-248.

[6] Planck, M. (1915) Das Prinzip der kleinstenWirkung. In (Planck, 1944), 68-78.

[7] Einstein, A. (1916) Hamiltonsches Prinzip und allgemeine Relativitätstheorie. Königlich Preußische Akademie der Wissenschaften (Berlin). Sitzungsberichte, 1111-1116.

[8] Feynman, R. http://liberzon.csl.illinois.edu/teaching/FeynmanLecturesOnPhysicsChapter2-19.pdf

[9] Mach, E. (1988) Die Mechanik in ihrer Entwicklung. Historisch-kritisch dargestellt. Wahsner, R. and von Borzeszkowski, H.-H., Eds., Akademie-Verlag, Berlin. (Authorized English translation: The Science of Mechanics. Account of 
Its Development, Open Court, La Salle, IL, 1989).

[10] Jacobi, C.G.J. (1866) Vorlesungenüber Dynamik. Ed. by A. Clebsch, Georg Reimer, Berlin.

[11] Zeilinger, A. (1999) Foundations of Physics, 29, 631;

Zeilinger, A. (2000) Nature, 408, 639. http://dx.doi.org/10.1038/35047177

[12] Tributsch, H., (2015) Irrationality in Nature or in Science? Probing a Rational Energy and Mind World. CreateSpace, a company of Amazon. 